Recepción: 20 / 04 / 2017

Aceptación: 20 / 05 / 2017

Publicación: 15 / 07 / 2017

Ciencias de la Salud

Artículo Científico

\title{
La flor Bunganvilla y su evaluación farmacognostica y preclínica como expectorante
}
The Buganvilla flower and its pharmacognostic and preclinical evaluation as expectorant
A flor Buganvílias e sua farmacognóstico e avaliação pré-clínica como um expectorante

\author{
Freddy A. Pereira-Guanuche ${ }^{\text {I }}$ \\ fpereira@utmachala.edu.ec \\ Kennya S. Ruiz-Veintimilla II \\ kruiz@utmachala.edu.ec \\ Kennya M. Pereira-Ruiz III \\ kpereira@utmachala.edu.ec
}

Correspondencia: fpereira@utmachala.edu.ec 


\section{Resumen}

El objetivo de la presente investigación es obtener por medio de los resultados la certificación de las propiedades que como expectorante tiene la flor de Buganvilla en el tratamiento de afecciones respiratorias (asma, bronquitis catarro, dolor de pecho, fiebre, gripe, pulmonía, ronquera, tos, tos ferina). Para ello se evaluaron los parámetros Farmacognósticos, Químicos Cualitativos y Cuantitativos siguiendo los procedimientos descritos en la literatura de Payo Amando (2001). Mediante un ensayo preclínico en ratas Wistar (machos), observada con una absorción obtenida con un espectrofotómetro ultravioleta de absorción lo cual confirmó que la flor si es expectorante. También se realizaron estudios Químico-Cualitativos indicando la presencia de Saponinas, Compuestos grasos, Triterpenos, Esteroides, Azúcares reductores, Compuestos Fenólicos, Flavonoides y Alcaloides ; con lo cual se concluyó que la flor Bougainvillea Glabra Choisy posee propiedades expectorantes debido a la presencia de los alcaloides cuya función es la de dilatar los bronquios posibilitando el aumento de la respiración.

Palabras Claves: Expectorante; buganvilla; respiratorias; afecciones; pulmonía. 


\section{Summary}

The objective of the present research is to obtain by means of the results the certification of the properties that as an expectorant has the flower of Buganvilla in the treatment of respiratory affections (asthma, cold bronchitis, chest pain, fever, flu, pneumonia, hoarseness, Cough, whooping cough). For this, the Pharmacognostic, Qualitative and Quantitative Chemical parameters were evaluated following the procedures described in the Payo Amando literature (2001). By means of a preclinical test in Wistar (male) rats, observed with an absorption obtained with an absorption ultraviolet spectrophotometer which confirmed that the flower is expectorant. Chemical-Qualitative studies were also performed indicating the presence of Saponins, Fatty Compounds, Triterpenes, Steroids, Reducing Sugars, Phenolic Compounds, Flavonoids and Alkaloids; With which it was concluded that the flower Bougainvillea Glabra Choisy possesses expectorant properties due to the presence of the alkaloids whose function is to dilate the bronchi enabling increased breathing.

Key words: Expectorant; buganvilla; respiratorias; affections; pneumonia. 


\section{Resumo}

O objectivo desta investigação é obtido através das propriedades resultados de certificação como um expectorante tem Bougainvillea flor no tratamento de desordens respiratórias (asma, bronquite catarro, dor no peito, febre, gripe, pneumonia, rouquidão, tosse, tosse convulsa). Para este farmacognostic, qualitativa e quantitativa de parâmetros químicos foram avaliados seguindo os procedimentos descritos na literatura Payo Amand (2001). Por um teste de pré-clínicos em ratos Wistar (macho), observado com uma absorção obtidos com um espectrofotómetro de absorção no ultravioleta o que confirmou que a flor se expectorante. Estudos bioquímicos foram realizadas qualitativa indicando a presença de saponinas, compostos gordos, triterpenos, esteróides, açúcares redutores, fenólicos, flavonóides e alcalóides; assim, concluiu-se que a flor Bougainvillea glabra Choisy tem propriedades expectorantes, devido à presença de alcalóides cuja função é dilatar os brônquios permitindo a respiração mais fácil.

Palavras-chave: expectorante, buganvílias, distúrbios respiratórios, pneumonia. 


\section{Introduccion,}

Según contaban los miembros mayores de edad de las comunidades nativas, antes de que llegaran los "blancos", el único medio de tratarse de cualquier mal eran las plantas medicinales, mediante mates, es decir hervido de las partes curativas para tomarlos oralmente, mediante baños tipo infusión emplastos, frotaciones externas en las partes afectadas, etc. Las hiervas o partes útiles de los árboles, generalmente los recogían los curanderos o shamanes, designados por los dioses y los colectaban de lugares escogidos, o sea no podían recolectar de cualquier lugar, sino de los más misteriosos, solo así podían tener efectos más curativos por sus principios activos y el don de los espíritus del bosque. Anyarin T. (2002)

Es por esta razón que se realizaron investigaciones ya que los científicos se vieron en la necesidad de entrar más a fondo en el estudio de las plantas con propiedades para sanar, curar y aliviar enfermedades por ejemplo en la ciudad de Machala se manifestó un grave problema como es el caso de las enfermedades respiratorias en donde la personas afectadas recurrieron a diferentes tipos de plantas medicinales que tuvieran actividad expectorantes que ejercieron sobre su organismo efectos beneficioso en especial sobre la fisiología del árbol bronquial a varios niveles: aumentándoles la producción de secreciones bronquio alveolares. Paz E. (1998)

Las enfermedades más comunes son: resfriado, gripe, bronquitis neumonía y pulmonía que afectan principalmente al tracto respiratorio manifestándose con una infección e inflamación tanto de la cavidad nasal, faringe, laringe, bronquios, y pulmón; estos problemas respiratorios son los causales más frecuentes de morbilidad y mortalidad tanto de niños, adultos y adultos mayores en la provincia de El Oro esto se debe a la contaminación ambiental, exposición al aire, cambios climáticos, y material particulado ( combustible quemado). 
Según la OMS la población con mayor riesgo de morir por ERA (Enfermedades respiratorias agudas) son los niños y niñas, las personas de tercera edad y las que tienen comprometido su sistema inmunológico. Las infecciones respiratorias superiores comúnmente conocidas como resfriado son muy frecuentes, pero rara vez han puesto en peligro la vida, en cambio las infecciones respiratorias bajas generalmente producidas por infecciones bacterianas, fueron responsables de cuadros más graves como la influenza, la neumonía y la bronquiolitis, las que contribuyen de forma importante a la mortalidad por ERA. En los países en desarrollo, se estimó que entre el 2 y el 3\% de los niños y niñas menores de dos años, tuvieron neumonía severa lo que requirió hospitalización y las tasas de mortalidad por IRA (Infecciones respiratorias agudas ) que se calcularon en estos países oscilaron entre 60 y 100 casos por 1.000 niños menores de cinco años. López. I. (2002)

Por tal motivo se seleccionó a la flor nativa del Brasil Buganvilla (Bougainvillea glabra Choisy) considerada como una planta medicinal con propiedad expectorante, en donde se evaluó científicamente algunos de los usos tradicionales y en un futuro ser utilizada en la elaboración de productos fitofármacos beneficiosos en personas que presentan infecciones respiratorias agudas y reducir las tasas de morbilidad y mortalidad en El Oro, El Ecuador y el mundo entero.

\section{Materiales y métodos.}

El diseño de investigación es no experimental; con estudio descriptivo, en el cual se describió las características de la Buganvilla (Bougainvillea glabra Choisy), del extracto acuoso, además se describieron métodos para su evaluación, elaboración, control de calidad y estudio experimental mediante ensayos pre-clínicos en animales de experimentación. 
Como métodos generales se empleó el método científico, inductivo y deductivo ya que se partirán de las hipótesis planteadas, las mismas que serán comprobadas mediante la investigación, llegando a una serie de conclusiones acerca del efecto que posee la planta en estudio, de lo cual surgieron las recomendaciones para el uso correcto de esta planta.

La presente investigación se realizó en la Planta Piloto de Farmacia de la Facultad de Ciencias Químicas y de la Salud de la Universidad Técnica de Machala, ubicada en el Km 5 y medio vía Pasaje, contando con condiciones de temperatura de $22-32^{\circ} \mathrm{C}$ y humedad de $62-82 \%$. Latitud: $3^{\circ} 16^{\prime}$ 0" S. Longitud:79 59' 0" W y altura 6 metros sobre el nivel del mar

Las muestra para la elaboración del extracto acuoso previa a la Evaluación Farmacognosia y preclínica fueron 2 de los 5 colores de flores existentes que presenta la especie Bougainvillea glabra Choisy seleccionados al azar de los 2 sectores de la ciudad de Machala específicamente en el barrio 3 de Noviembre y ciudadela los Vergeles considerados en la realización de este trabajo.

\section{Resultados}

De los 25 consumidores de plantas medicinales encuestados el $80 \%$ de ellos manifiesta tener una preferencia por la flor Bouganvilla Glabra Choisy para el tratamiento de la tos, mientras que el 64 $\%$ de ellos prefiere a la manzanilla y menta, seguida la Borraja con el 56\%, la Flor de Violeta y el cedrón con un $36 \%$, Flor de tilo con un $32 \%$, Anís con un 24\%, la Pulmonaria con el $16 \%$, la Hierba Luisa , Canela .Orégano y la Ortalisa con un $8 \%$ y la Ruda Hoja de Naranjo y la Uña de gato con el $4 \%$.

Para la perdida por desecación el valor que se obtuvo es del 7,9 \%, el mismo que se encuentra dentro de los parámetros referenciales de la farmacopea de los estados Unidos de América (2007). 
Los valores de las cenizas totales se encuentran elevados con respecto a los reportados en la literatura mostrando hasta un máximo del $12 \%$, lo mismo que las cenizas solubles en agua e insolubles en $\mathrm{HCl}$, por lo que sugiere que el tipo de suelo en donde crece la planta en estudio es rico en minerales.

Los resultados de las sustancias solubles en las diferentes soluciones son, para el Alcohol de $70^{\circ}$ es de $9,80 \%$, y alcohol de $50^{\circ}$ es de $5,79 \%$ por lo q se demuestra que en este último la extracción del alcohol de 50 es más bajo que el de 70 y para el agua es del 6,72 \%, mostrando una mejor extracción el solvente alcohólico de $70^{\circ}$.

Con lo que respecta a la desviación estándar de cada uno de los porcentajes de los ensayos no difieren en su promedio final es decir la pérdida de porcentaje es mínima como por ejemplo:

El porcentaje de efectividad de la flor Bougainvillea Glabra Choisy es de 25,625\% lo que me indica que se logró llegar al efecto deseado de la investigación ya que esta cantidad se aproxima al $31.87 \%$ de la Bromexhina que es la muestra patrón que se utilizó como guía en el ensayo

\section{Conclusiones}

Se Determinaron las cualidades físicas y químicas de la especie de la flor Bougainvellea Glabra Choisy mediante la especificaciones de calidad observando sus características cualitativas por medio de métodos y equipos utilizados para los ensayos farmacognósticos y preclínicos. Realizando asi las respectivas inspecciones por lo que se se concluyó que la especie esta apta para el consumo de los seres humanos ya que esta en las respectivas condiciones físicoquímicas ynocausadañosparalasalud.

- La flor Bougainvillea Glabra Choisy si posee principios activos como saponinas, compuestos grasos, triterpenos, esteroides, azúcares reductores, compuestos fenólicos, flavonoides 
y alcaloides y estos son los que contribuyen a que la planta realice el principal efecto farmacológico que buscaba en esta investigación la cual consiste en hacer fluir la mucosidad presente en los pulmones, tráquea y bronquios de los seres humanos es decir todo lo que respecta al sistema respiratorio que lo comprobé mediante un ensayo in vivo .

- $\quad$ En La flor bougainvillea Glabra Choiry encontré a través del método por espectofotometría de la luz ultravioleta la presencia de alcaloides principalmente en sus brácteas tal como lo indicaba la literatura y para lo cual investigue que estos compuestos químicos son necesarios para que los pulmones se dilaten y asi el flujo pueda salir del aparato respiratorio.

- A través de un ensayo preclínico demostré que la ESPECIE si presentaba la propiedad de expectorar ya que por medio de un extracto de la flor bougainvillea glabra choisy administrada en ratas de laboratorio, extrayendo luego una parte de su tráquea que diluida en $1 \mathrm{ml}$ de suero fisiológico le medí la absorbancia de mucosa en un equipo llamado absorbancia espectrofotómetro de ultravioleta que confirmó que la mucosidad si se adhería a la tráquea de las ratas

- La planta podrá ser utilizada en un futuro para la fabricación de nuevos productos fitofármacos ya que al realizarle los respectivos ensayos farmacognósticos demostraron que la especie posee actividad expectorante en patologías respiratorias.

- $\quad$ El género más frecuente fue el femenino con mayor mortalidad para NAH y NAC, habiendo mayor morbilidad en NAC para los hombres, en la mortalidad hospitalaria el género femenino es 1,5 veces mas que el masculino. La estancia hospitalaria fue mayor en los pacientes hospitalizados por NAH. La edad promedio para NAC fue de 62 y de 61 años para NAH. El factor de riesgo más frecuente fue hipertensión arterial seguido de diabetes mellitus, insuficiencia renal cónica, enfermedad cardíaca crónica y enfermedad cerebro vascular entre las más importantes, sin embargo, 
es importante recordar que los pacientes que son renales crónicos son diabéticos y en su mayoría hipertensos en este estudio, además la mayoría de los pacientes se encontraban en tratamiento de sustitución renal ( diálisis o diálisis peritoneal ), lo que haría que este grupo de pacientes tengan una connotación especial, esta se relacionó con mayor morbilidad y la ECV con mayor mortalidad.

- La causa de ingreso se relacionó con aspiración, convulsiones, enfermedad cerebro vascular aguda y convulsiones; en general, deterioro del nivel de consciencia que facilita la colonización de bacterias en la oro faringe, el que más se relacionó con mortalidad fue el ECV. El uso de antibióticos fue mayor en los pacientes con NAH y con mayor mortalidad, casi el doble de pacientes traqueotomizados para los pacientes con NAC y mayor mortalidad; la cirugía abdominal fue la responsable de mayor morbimortalidad en ambos grupos, la cirugía ortopédica mayor mortalidad en los pacientes que tuvieron NAH.

- $\quad$ En las complicaciones la sepsis presentó 4 veces más pacientes con NAH que los de NAC se asoció con mayor morbilidad, el shock séptico se relacionó con mayor mortalidad en los pacientes con NAH.

- $\quad$ El agente causal más frecuente fue la Klebsiellapneumoniae con una morbilidad dos veces a uno en relación a la mortalidad, la Pseudomonaaeuriginosa con mayor mortalidad en los pacientes con NAC y mayor morbilidad de los pacientes con NAH, mayor mortalidad que morbilidad de los pacientes que tuvieron Acinetobacterbaumanii; hubo mayor sensibilidad a los carbapenems en los pacientes con NAC que NAH generando mayor mortalidad, alta resistencia para las cefalosporinas, las penicilinas con un beta-lactámico tuvieron una aceptable sensibilidad, muy poca resistencia sobre vancomicina en los pacientes con Staphylococcussureus; a pesar de que la colistinatinen valores bajos en el presente estudio es necesario tener en cuenta que era sensible en la mayoría de los pacientes con resistencia a los carbapenems, aunque en muy pocos pacientes estuvo reportado 
resistencia para la colistina, lo que sugiere un problema grave para el tratamiento de los pacientes severamente enfermos.

\section{Recomendaciones}

- $\quad$ En el momento que se realiza el Tamizaje fitoquímico de la flor Bougainvillea Glabra Choysi al molerla se pueden obtener sólidos y si así fuera el caso se recomienda hacerlo preparando un extracto diluido de 1:10 para que estas partículas no interfieren en la apreciación de la formación de precipitados y cambios de coloración.

- Al momento de hacer la extracción de la flor ya sea en agua o en alcohol se recomienda utilizar el equipo de extracción al vació ya que el polvo que se obtiene de después de pulverizar es muy fino y por consecuente absorbe más cantidad de líquido.

- Realizar la cuantificación de los metabolitos encontrados mediante el estudio químico cualitativo para elaborar una formulación que permita obtener un producto de calidad.

\section{Bibliografía.}

Alonso Paz E. (1998, Julio)Edición CETAAR - INCUPO Uso racional de plantas medicinales. Revista Farmacéutica. Consultado el 15 de febrero, 2013. http://www.angelfire.com/ar/plantasmedicinales/monograf1.html salud.es revista

Álvarez J. Medellin R. Rattusnorvegicus. Febrero 2005. Universidad Nacional Autónoma de México. Bases de datos SNIB-CONABIO. Proyecto U020. México. D.F. Disponible en: http://www.conabio.gob.mx/conocimiento/exoticas/fichaexoticas/Rattusnorvegicus00.pdfConsultado: 13/Diciembre/2012

Anyarin t. (2002). Plantas Medicinales Nº1 Lima: Una OrganizaciónParuana al Servicio de la Cultura y el sano entretenimiento.

Arango G.: Alcaloides y Compuestos Nitrogenados. Universidad de Antioquia. Medellín, Colombia (2008).

Berkow, R. (1899). MANUAL MERRCK DE INFORMACIÓN MÉDICA PARA EL HOGAR: Océano, S.A. España: Océano grupo editorial.

Cáceres, A. (1996). Plantas de Uso Medicinal en Guatemala: Primera Edición en editorial Universitaria. Guatemala: Universidad de San Carlos Guatemala. 
EnglerH, Szelenyi I (1984) Tracheal phenol red secretion, a new method for screening muco secretolytic compounds, J Pharmacol Meth 11:151 - 157

Font, E. (2002, Diciembre 11). Etiología, diagnóstico, profilaxis y tratamiento del resfriado común. ELSEVIER. 21. Consultado el 3 de Septiembre del 2013. En:http://zl.elsevier.es/es/revista/offarm4/etiologia-diagnostico-profilaxis-tratamiento-resfriado-comun-13041298 ambito_farmaceuticoeducacionsanitaria-2002

Goodman A., Goodman I., Gilman A. (1980) The Pharmacological basis of the Therapeutic 6 ed Mac Millan Public, Co. Inc. N.Y. pag 960

León J. 2011. Efecto Hipoglucemiante del Extracto de las Hojas de Frutipan (Artocarpusaltilis) en Ratas con Hiperglucemia Inducida. Escuela Superior Politécnica de Chimborazo. Riobamba - Ecuador.

López. I. (2002) Alergia e Inmunología. Libro Originador: Centro Nacional de Información de Ciencias Médicas. Editorial Ciencias Médicas Fuente: Depto. de Epidemiología Ministerio de Salud Manual control de infección Respiratorias. [En Línea].Consultado:(23 de Febrero, 2013) En: http:// www.portalesmedicos.com/.../Infecciones-Respiratorias-Agudas.

Payo Amando (2001) tamizaje fotoquímico preliminar de especies del género crotón instituto de ecología y sistemática Rev. Cubana Farm; 35(3):203-206 Cuba.

Sánchez, J. J. (2012, Septiembre1). Bien Contigo-Salud.Las enfermedades respiratorias generalmente son ocasionadas por microorganismos o sustancias tóxicas presentes en el ambiente. Extraído el 1 de Septiembre del 2013 desdehttp://www.esmas.com/salud/home/conocetucuerpo/363910.html

Washington J. (2006, Octubre 12) Las enfermedades respiratorias: resfriado, gripe, bronquitis y neumonía/pulmonía SALUD CULTURA. 74 Consultado (3 de Marzo del 2013) En: http://www.saluddealtura 